\title{
Thirty-day Outcomes of On-Pump and Off-Pump Coronary Artery Bypass Grafting: an Analysis of a Brazilian Sample by Propensity Score Matching
}

Álvaro Rösler¹, BMSc, MSc; Gabriel Constantin1, BMSc; Pedro Nectoux¹, BMSc; Bruno Sell Holz¹, MD; Estevan Letti ${ }^{1}$, MD; Marcela Sales ${ }^{1}$, MD, MSc; Fernanda Lucchese-Lobato ${ }^{2}$, PhD; Fernando Lucchese ${ }^{1,2}$, MD

\begin{abstract}
Introduction: Coronary artery bypass grafting (CABG) performed with and without cardiopulmonary bypass (CPB) support has been widely discussed in the literature. However, little is known about the outcomes of those techniques in Brazil. This study aims at exploring 30-day mortality and morbidity outcomes of on- and off-pump isolated CABG in a large sample from Southern Brazil.

Methods: A single-center cohort with 1,767 patients undergoing isolated CABG (January 2013 - December 2018) was initially evaluated. Patients undergoing off-pump ( $N=397)$ and on-pump $(N=1,370)$ CABG were identified. To obtain two completely homogeneous study groups, propensity score matching was used. The paired groups were compared by descriptive and univariate analyses. Then, logistic regression was used to verify the effects of on- and off-pump CABG on 30-day mortality.
\end{abstract}

Results: None of the baseline characteristics showed significant difference between the groups $(P>0.05)$. None of the analyzed morbidity outcomes showed any difference between the groups, including acute myocardial infarction $(3.0 \%$ vs. $1.5 \% ; P=0.192)$, stroke $(2.4 \%$ vs. $4.2 \% ; P=0.193)$, and major reoperation $(0.6 \%$ vs. $0.3 \% ; P=1.000$ ), as well as the major adverse cardiovascular and cerebrovascular events composite outcome $(6.3 \%$ vs. $7.5 \%$; $P=0.541)$. Mortality also did not differ ( $1.5 \%$ vs. $2.4 \% ; P=0.401)$, and $C P B$ support was not an independent predictor of risk for 30-day mortality (odds ratio: 2.052 ; $95 \%$ confidence interval: $0,609-6.913 ; P=0.246$ ).

Conclusion: After matching by propensity analyses, similar rates of on- and off-pump 30-day mortality and other major outcomes were observed. In addition, the use of CPB support was not an independent predictor of risk for the occurrence of 30-day mortality.

Keywords: Cardiopulmonary Bypass. Morbidity. Propensity Score. Logistic Model. Reoperation, Myocardial Infarction.

\begin{tabular}{llll}
\hline Abbreviations, acronyms \& symbols & & \\
\hline AF & = Atrial fibrillation & LV & $=$ Left ventricle \\
AMI & = Acute myocardial infarction & MACCE & $=$ Major adverse cardiovascular and cerebrovascular events \\
B & = Unstandardized regression weight. & NYHA & $=$ New York Heart Association \\
CABG & $=$ Coronary artery bypass grafting & OR & $=$ Odds ratio \\
CI & $=$ Confidence interval & PASP & $=$ Pulmonary artery systolic pressure \\
COPD & $=$ Chronic obstructive pulmonary disease & PCI & $=$ Percutaneous intervention \\
CPB & $=$ Cardiopulmonary bypass & PVD & $=$ Peripheral vascular disease \\
CV & $=$ Cardiovascular & RCTs & $=$ Randomized clinical trials \\
EuroSCORE & European System for Cardiac Operative Risk Evaluation & SE & $=$ Standard error \\
HF & $=$ Heart failure & STS & $=$ Society of Thoracic Surgeons
\end{tabular}

${ }^{1}$ Research Center of Cardiovascular Surgery, São Francisco Hospital, Santa Casa de Misericórdia de Porto Alegre, Porto Alegre, Rio Grande do Sul, Brazil.

²Pediatric brain-heart Clinic, Santo Antônio Hospital, Santa Casa de Misericórdia de Porto Alegre, Porto Alegre, Rio Grande do Sul, Brazil.

This study was carried out at the Hospital São Francisco, Santa Casa de Misericórdia de Porto Alegre, Porto Alegre, Rio Grande do Sul, Brazil.
Correspondence Address:

Álvaro Rösler

(iD) https://orcid.org/0000-0003-4727-3367

Centro de Pesquisas em Cirurgia Cardiovascular

Hospital São Francisco - Santa Casa de Misericórdia de Porto Alegre

Rua Prof. Annes Dias, 285, Centro Médico do Hospital São Francisco, Térreo, Bairro Centro, Porto Alegre, RS, Brasil - Zip Code: 90020-090

E-mail: alvaromrosler@gmail.com 


\section{INTRODUCTION}

In Brazil, coronary disease is a condition with very high prevalence ${ }^{[1]}$. The standard treatment for complex coronary disease is coronary artery bypass grafting $(C A B G)^{[2-5]}$. Coronary surgery was introduced in Brazil by Drs. Jatene and Zerbini in late 1960 s and is the most widely performed surgical cardiovascular procedure in Brazil. In fact, more than 20,000 surgical revascularizations are performed every year, representing 113 CABGs per million inhabitants in Brazil annually ${ }^{[1]}$. CABG patients in Brazil have high prevalence of several cardiovascular risk factors, and the national mortality rates are about $6 \%{ }^{[6]}$.

Worldwide, the question regarding the effectiveness of cardiopulmonary bypass (CPB) support has become debatable and even controversial. Randomized clinical trials (RCTs) comparing on and off CPB techniques have shown mixed results ${ }^{[7-10]}$. Thus, a scientific consensus is yet to be reached on the best practice for CPB support use in $\mathrm{CABG}^{[12]}$. Very few studies have compared both techniques in Brazilian samples, and no large-scale RCT has been done in Brazil with the same aim ${ }^{[12-14]}$.

In Brazil, controlled RCTs of cardiovascular surgeries are often not performed due to a dearth of clinical research teams in most cardiovascular centers, as well as lack of research funding. Recently, an emergent statistical methodology has been increasingly used to compare interventions by using observational data from cohorts. For instance, the propensity score matching analysis has become a feasible and powerful approach to study surgical outcome data ${ }^{[10,15-17]}$ without the costs of doing an RCT, while still controlling for heterogeneity in the sample.

This study aims at using a propensity score matching analysis to compare the outcomes of two groups of post isolated CABG patients (on- vs. off-pump) in a reference cardiovascular center in Southern Brazil.

\section{METHODS}

This study protocol received full approval from the institutional ethics review board (2.006.177) and departmental research committee. It complies with the ethical guidelines of the Declaration of Helsinki. As this is a retrospective observational study of clinical surgical practice, the consent form was not required by the local committee.

We analyzed a single-center cohort with 1,767 patients who underwent isolated CABG between January 2013 and December 2018. Of these surgeries, 397 (22.5\%) were performed with offpump technique, and 1,370 (77.5\%) were performed with onpump technique. A standard median sternotomy was performed in all patients. As an uncontrolled cohort study, the criteria used to choose the surgical technique was subjective and dependent on each surgeon's discernment.

Sample heterogeneity is often observed in randomized controlled trials, and this study used a propensity score matching analysis by a logistic regression mode ${ }^{[18]}$ to obtain two completely homogeneous comparison groups. A logistic regression model was built with the categorical variable of CPB support as the dependent variable. The independent variables were 21 baseline and clinical characteristics including gender, age, weight, hypertension, diabetes, acute myocardial infarction (AMI), renal impairment, hemodialysis, smoking, chronic obstructive pulmonary disease (COPD), pulmonary artery systolic pressure (PASP), stroke, peripheral artery disease, atrial fibrillation (AF), New York Heart Association class III or IV heart failure, frailty, anemia, instable angina, previous cardiovascular surgery, stenosis $>50 \%$ in the left main coronary artery, and emergency surgery.

The probabilities generated for each patient were used as scores to establish the best match. To form a pair, it was necessary to have the same value in the three first decimals. The fourth decimal being the tiebreaker criterion in the pairing. This way, it was possible to obtain 332 pairs ( $N=664)$ of very similar patients. In Figure 1, the equality of the propensity score matching values between the two intervention groups is presented.

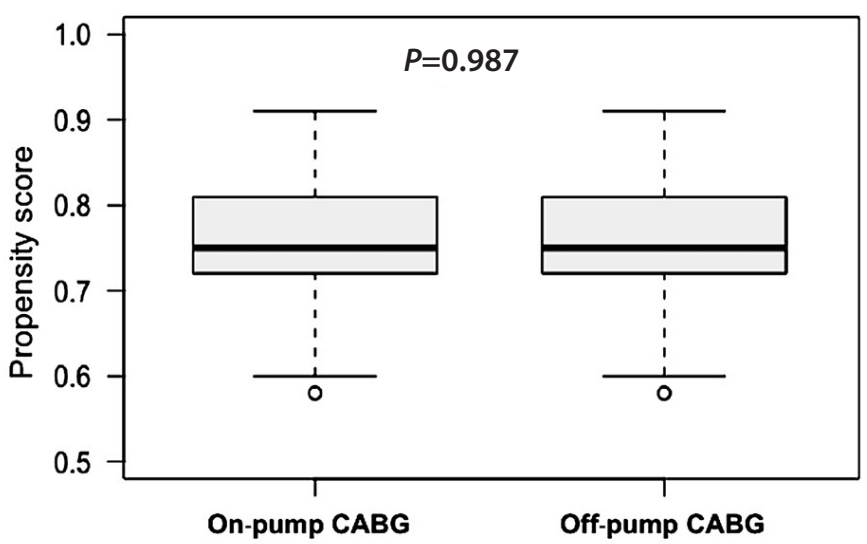

Fig. 1 - Boxplot of the propensity scores of stratified paired study groups. $C A B G=$ coronary artery bypass grafting.

After the propensity score matching, we performed normality analyses for all quantitative variables evaluated in the study - age, creatinine clearance, left ventricular ejection fraction, PASP, European System for Cardiac Operative Risk Evaluation (EurOSCORE) I, EuroSCORE II, and Society of Thoracic Surgeons (STS) score. The distribution pattern was evaluated by skewness and kurtosis coefficients and Kolmogorov-Smirnov test. Coefficients between -3 and +3 and a Kolmogorov-Smirnov test $P$-value $>0,05$ indicated a normal distribution. In this way, EurOSCORE I, EurOSCORE II, and STS score presented asymmetric distributions and were analyzed by univariate non-parametric Mann-Whitney $U$ test. Other quantitative variables with normal distributions were analyzed with $t$-test for independent samples. Descriptions of the quantitative variables were made by mean and standard deviation. Qualitative variables were described by absolute number and the related proportion (\%). To analyze this kind of variable we applied the two tailed Pearson's Chi-square.

The baseline characteristics and outcomes were compared according to the study group (on-pump vs. off-pump) with the univariate tests previously mentioned. For this analysis, a $P$-value $<0.05$ was considered significant. A univariate analysis stratified by the occurrence of death in 30 days was also carried out in order to evaluate and select potential predictors for the 
occurrence of the outcome. Only in this case, to select the independent variables for the regression model, we considered significant $P$-values $<0.10$ in the analysis stratified by death rates.

Based on the univariate analysis, we selected for the regression model the following variables as independent variables: gender, COPD, AF, and preoperative anemia. In addition to these variables, we also used CPB as an independent variable because it is our main stratification variable. Therefore, the regression model had, as a dependent variable, the occurrence of death in 30 days and, as independent variables, gender, COPD, AF, preoperative anemia, and use of CPB. Bearing in mind that our outcome is a dichotomous categorical variable, we used the corresponding multivariate model for the analysis, a binary logistic regression.

\section{RESULTS}

None of the baseline clinical and demographic characteristics showed a significant difference between the groups (Table 1). This demonstrates a high degree of homogeneity between the two groups, obtained through propensity matching technique,

Table 1. Baseline characteristics stratified by CPB support.

\begin{tabular}{|c|c|c|c|}
\hline Characteristics & $\begin{array}{l}\text { Off-pump CABG } \\
\qquad(n=332)\end{array}$ & $\begin{array}{l}\text { On-pump CABG } \\
\qquad(n=332)\end{array}$ & $P$-value \\
\hline Female gender & $98(29.5 \%)$ & $108(32.5 \%)$ & 0.402 \\
\hline Age (years) & $62,7 \pm 9,8$ & $62,3 \pm 8,6$ & 0.568 \\
\hline Hypertension & $276(83.1 \%)$ & $270(81.3 \%)$ & 0.542 \\
\hline Diabetes & $128(38.6 \%)$ & $138(41.6 \%)$ & 0.428 \\
\hline AMl & $109(32.8 \%)$ & $109(32.8 \%)$ & 1.000 \\
\hline Renal impairment & $32(9.6 \%)$ & $33(9.9 \%)$ & 0.986 \\
\hline Hemodialysis & $08(2.4 \%)$ & $12(3.6 \%)$ & 0.364 \\
\hline Creatinine clearance & $75,1 \pm 27,2$ & $77,3 \pm 29,9$ & 0,333 \\
\hline Smoking & $67(20.2 \%)$ & $67(20.2 \%)$ & 1.000 \\
\hline COPD & $16(4.8 \%)$ & $18(5.4 \%)$ & 0.725 \\
\hline Stroke & $25(7.5 \%)$ & $24(7.2 \%)$ & 0.882 \\
\hline PVD & $14(4.2 \%)$ & $13(3.9 \%)$ & 0.844 \\
\hline Atrial fibrillation & $08(2.4 \%)$ & $07(2.1 \%)$ & 0.794 \\
\hline NYHA class III or IV HF & $63(19 \%)$ & $61(18.4 \%)$ & 0.842 \\
\hline LV ejection fraction (\%) & $61 \pm 12,0$ & $60 \pm 12,7$ & 0.199 \\
\hline PASP (mmHg) & $29,2 \pm 6,6$ & $29,3 \pm 7,6$ & 0.957 \\
\hline Frailty & $27(8.1 \%)$ & $26(7.8 \%)$ & 0.886 \\
\hline Anemia & $99(29.8 \%)$ & $97(29.2 \%)$ & 0.865 \\
\hline Instable angina & $24(7.2 \%)$ & $31(9.3 \%)$ & 0.324 \\
\hline Previous CV surgery & $6(1.8 \%)$ & $3(0.9 \%)$ & 0.505 \\
\hline Previous PCl & $82(24.7 \%)$ & $62(18.7 \%)$ & 0.060 \\
\hline Urgency or emergency & $9(2.7 \%)$ & $7(2.1 \%)$ & 0.613 \\
\hline EuroSCORE I & $3.34 \pm 4.24$ & $3.26 \pm 3.34$ & 0.805 \\
\hline EuroSCORE II & $1.56 \pm 1.71$ & $1.54 \pm 1.07$ & 0.808 \\
\hline STS score & $0.98 \pm 1.01$ & $1.08 \pm 1.07$ & 0.202 \\
\hline Complete revascularization & $321(96.7 \%)$ & $320(96.4 \%)$ & 0.832 \\
\hline
\end{tabular}

$\mathrm{AMI}=$ acute myocardial infarction; $\mathrm{CABG}=$ coronary artery bypass grafting; $C O P D=$ chronic obstructive pulmonary disease; $\mathrm{CPB}=$ cardiopulmonary bypass; $\mathrm{CV}=$ cardiovascular; EuroSCORE=European System for Cardiac Operative Risk Evaluation; HF=heart failure; LV=left ventricular; NYHA=New York Heart Association; PASP=pulmonary artery systolic pressure; $P C I=$ percutaneous intervention; PVD=peripheral vascular disease; STS=Society of Thoracic Surgeons 
allowing for a solid comparison between the 30-day outcomes of isolated $C A B G$.

None of the analyzed outcomes showed any differences between the groups, including AMI (3.0\% vs. 1.5\%; $P=0.192)$, stroke ( $2.4 \%$ vs. $4.2 \%$; $P=0.193)$, major reoperation ( $0.6 \%$ vs. $0.3 \%$; $P=1,000)$, major adverse cardiovascular and cerebrovascular events (MACCE) (6.3\% vs. 7.5\%; $P=0.541)$, and death (1.5\% vs. $2.4 \% ; P=0.401)$. All the major outcome comparisons are shown in Figure 2. The overall mortality rate was $2.0 \%$.

It was possible to establish, through regression analyses, that the use of CPB was not an independent predictor of risk for the occurrence of death (odds ratio [OR]: 2.052; 95\% confidence interval $[\mathrm{Cl}]$ : $0,609-6.913 ; P=0.246)$. Furthermore, other variables with univariate association with 30-day mortality were independent predictors for the outcomes - gender (OR: 4.659, 95\% Cl: $1.375-15.787 ; P=0.013)$, COPD (OR: 5.903, 95\% Cl: 1.316 - 26.469; $P=0.020$ ), preoperative AF (OR: 9.550, 95\% Cl: 1.507 60.509; $P=0.017$ ), and preoperative anemia (OR: $4.150,95 \% \mathrm{Cl}$ : $1.272-13.541 ; P=0.018$ ) (Table 2).

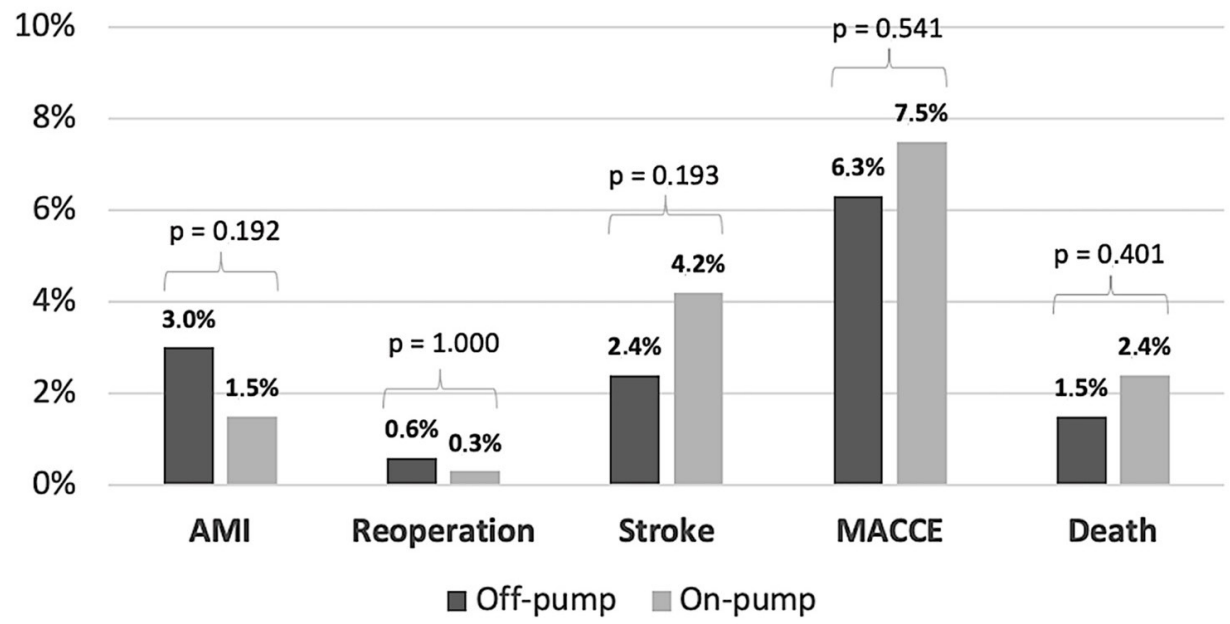

Fig. 2 - Thirty-day outcomes rates stratified by cardiopulmonary bypass support. AMI=acute myocardial infarction; MACCE=major adverse cardiovascular and cerebrovascular events.

\section{DISCUSSION}

This study adds to our knowledge on the outcomes of onvs. off-pump CABG procedures in Southern Brazil. It shows that there are no significant differences between on- and off-pump CABG mortality and morbidity. In addition, a logistic regression adjusted model did not predict that CPB support is a risk factor for 30-day post-surgery mortality rates in this sample.

These findings are congruent with findings from Lamy et al. ${ }^{[9]}$ (2012) that showed no differences between on- and off-pump isolated CABG mortality and morbidity outcomes within the CORONARY trial study, an international multicenter randomized controlled trial. Mortality rates in the CORONARY trial study were of $2.5 \%$ in both on- and off-pump groups, which was similar to our study, especially in the on-pump group, however our offpump group showed slightly lower rates of mortality (1.5\%).

Another large multicenter randomized controlled trial study, called ROOBY trial ${ }^{[8]}$, also showed a lack of significant differences between on- and off-pump CABG procedures. Like in the CORONARY trial, this trend was similar to our results, however, the 30-day mortality rates observed in the ROOBY trials were higher than those observed in our study $(7 \%$ off-pump and 5.6\% on-pump).

The observed differences in mortality rates may be due to the large number of centers involved in data collection and possible heterogeneity in training among the surgeons who performed the surgeries in those large trials ${ }^{[7-9]}$. For instance, the present study involved only one center, with four very experienced surgeons (an average of 15 years of experience for both techniques), who had previously performed at least 250 surgeries of each procedure (onand off-pump).

Table 2. Logistic regression analysis.

\begin{tabular}{l|c|c|c|c|c|c}
\hline \multicolumn{1}{c|}{ Variables } & B & SE & Wald & P & OR & 95\% CI \\
\hline Gender (male) & 1.539 & 0.623 & 6.109 & 0.013 & 4.659 & $1.375-15.787$ \\
\hline COPD (yes) & 1.775 & 0.766 & 5.378 & 0.020 & 5.903 & $1.316-26.469$ \\
\hline Atrial fibrillation (yes) & 2.257 & 0.942 & 5.738 & 0.017 & 9.550 & $1.507-60.509$ \\
\hline Anemia (yes) & 1.423 & 0.603 & 5.563 & 0.018 & 4.150 & $1.272-13.541$ \\
\hline CPB (yes) & 0.719 & 0.620 & 1.345 & 0.246 & 2.052 & $0.609-6.913$ \\
\hline Constant & -6.050 & 0.826 & 53.586 & $<0.001$ & 0.002 & $\ldots$. \\
\hline
\end{tabular}

$\mathrm{B}=$ unstandardized regression weight; $\mathrm{Cl}=$ confidence interval; $\mathrm{COPD}=$ chronic obstructive pulmonary disease; $\mathrm{CPB}=$ cardiopulmonary bypass; $\mathrm{OR}=$ odds ratio; $\mathrm{SE}=$ standard error 
Large randomized controlled trials are very difficult to be done, especially in developing nations, with limited research resources, like Brazil. The propensity matching score model allows for retrospective analyses of surgical outcome data that can be compared to RCT results done in large multicenter studies. Previous studies using propensity matching scores in CABG outcome data have been successful in retroactively evaluating mortality and morbidity in on- and off-pump CABG outcomes ${ }^{[15,16]}$.

While Bakaeen's ${ }^{[19]}$ study had a similar rate of off-pump procedure (18-24\%) as compared to our study rates of off-pump surgery (22.5\%), Brewer et al. ${ }^{[15]}$ showed lower rates of off-pump procedures (9\%). These studies analyzed 1:1 matching of both procedures and indicated similar results. These results highlight that our center in Southern Brazil has produced similar outcomes as international studies in terms of mortality and morbidity, while using a propensity matching score. Our results are relevant as they shed light on Brazilian CABG outcomes in a large scale.

On the other hand, in a cohort of the State of New York (United States of America) with 49,830 patients, a proportion of off-pump surgeries of $27.8 \%$ was verified. It was slightly higher than that verified in our study. Using a methodology that used propensity score matching, the researchers found significantly lower rates of mortality and complications associated with shortterm off-pump surgery ${ }^{[20]}$.

In the past, we had few Brazilian studies comparing on- and off-pump CABG surgeries. In 2004, Lima et al. ${ }^{[12]}$ published the results of an analysis of 73 Brazilian octogenarian patients. The researchers observed for on-pump CABG patients a surgical mortality rate equal to $11.5 \%$, while the off-pump patients had a surgical mortality rate equal $2.1 \%$. In another study by the same group of researchers, Sá et al. ${ }^{[13]}$ (2010) showed the results of onand off-pump CABG in a cohort with 941 women. The surgical mortality rate for off-pump CABG was lower when compared with the on-pump technique (3.1\% vs. 5.3\%), but without statistical significance. These two studies were performed considering specific patient characteristics (age or gender), while our study encompassed all patients of the center. Thus, both studies have important information about Brazilian patients, and demonstrated different trends in relation to mortality compared to our study - e.g., lower mortality rate for off-pump CABG, compared to on-pump. In 2012, Cantero et al. ${ }^{[14]}$ published the results of a comparison between the two techniques using a cohort with 177 patients. The researchers verified that the mortality rate was similar between the techniques. However, postoperative AMl rates were higher in the on-pump CABG group (7.6\%, off-pump; $12.9 \%$, on-pump). In our study, we did not observe this same pattern, and, in addition, AMl rates were much lower (3.0\%, off-pump; $1.5 \%$, on-pump).

Finally, we were able to match 664 patients and obtained two very similar groups. In this way, it was possible to compare the 30-day outcomes for the two surgical techniques of revascularization (off-pump and on-pump) more effectively. The propensity score matching is a way of emulating a randomization process, and it raises the level of evidence generated through a cohort study. Our initial cohort had 1,767 patients, and, with the matching, 1,103 patients were discarded from the analysis. This reduction in the number of individuals in the sample is part of the strategy of the propensity score matching, in which a larger number of participants is neglected so that very similar pairs of patients are formed. We think that the confounding results from different studies on the outcomes of the two techniques remains, thus more studies with similar methodologies or RCTs are needed in different populations, as our results contribute to shed light on the characteristics of our population and on the surgical results obtained with CABG procedures in Brazil.

\section{Limitations}

Although all patients were operated on by the same group of surgeons and underwent the same pre and postoperative care protocols, the study was carried out at a single institution. Thus, it is likely that our study represents, in some way, only the population of Southern Brazil. Due to the vast Brazilian territory, there are several different regions, and, as a result, we have an important heterogeneity in healthcare structures and also in the prevalence of cardiovascular risk factors. Another important point, addressed at the end of the discussion, is the reduction in the number of individuals in the analysis with the application of propensity score matching, which may neglect patients with unique characteristics. However, this is a necessary action for obtaining fully balanced study groups. In this way, the comparison of outcome rates can be performed more safely. However, even with the use of this statistical technique that makes the evidence generated from a cohort more reliable and robust, the study does not have the level of evidence from an RCT.

\section{CONCLUSION}

After analysis by propensity score matching, it was possible to observe that patients who underwent surgery with and without CPB had similar incidences of mortality, AMI, stroke, major reoperation, and MACCE in the 30 days post-CABG. It was also possible to verify that the use of CPB was not an independent risk predictor for the occurrence of 30-day mortality. In addition, we could observe that gender, COPD, preoperative $A F$, and preoperative anemia were independent risk predictors for the occurrence of post-CABG 30-day mortality.

\section{No financial support. \\ No conflict of interest.}

\section{REFERENCES}

1. Oliveira EL, Westphal GA, Mastroeni MF. Demographic and clinical characteristics of patients undergoing coronary artery bypass graft surgery and their relation to mortality. Rev Bras Cir Cardiovasc. 2012;27(1):52-60. doi:10.5935/1678-9741.20120009.

2. Cundiff DK. Coronary artery bypass grafting (CABG): reassessing efficacy, safety, and cost. MedGenMed. 2002;4(2):7.

3. Taggart DP. Best practices in coronary revascularization procedures: are we where we should be? Curr Opin Cardiol. 2014;29(6):528-33. doi:10.1097/HCO.0000000000000111. 
4. Squiers JJ, Mack MJ. Coronary artery bypass grafting-fifty years of quality initiatives since Favaloro. Ann Cardiothorac Surg. 2018;7(4):516-20. doi:10.21037/acs.2018.05.13.

5. Paez RP, Hossne Junior NA, Santo JADE, Berwanger O, Santos RHN, Kalil RAK, et al. Coronary artery bypass surgery in Brazil: analysis of the national reality through the BYPASS registry. Braz J Cardiovasc Surg. 2019;34(2):142-8. doi:10.21470/1678-9741-2018-0313.

6. Kaufman R, Kuschnir MCC, Xavier RMA, Santos MA, Chaves RrBM, Müller $R E$, et al. [Epidemiological profile for coronary artery bypass grafting surgery]. Rev Bras Cardiol. 2011;24(6):369-76. Portuguese.

7. Novitzky D, Shroyer AL, Collins JF, McDonald GO, Lucke J, Hattler B, et al. A study design to assess the safety and efficacy of on-pump versus off-pump coronary bypass grafting: the ROOBY trial. Clin Trials. 2007:4(1):81-91. doi:10.1177/1740774506075859.

8. Shroyer AL, Grover FL, Hattler B, Collins JF, McDonald GO, Kozora E, et al. On-pump versus off-pump coronary-artery bypass surgery. N Engl J Med. 2009:361(19):1827-37. doi:10.1056/NEJMoa0902905.

9. Lamy A, Devereaux PJ, Prabhakaran D, Taggart DP, Hu S, Paolasso E, et al. Off-pump or on-pump coronary-artery bypass grafting at 30 days. N Engl J Med. 2012;366(16):1489-97. doi:10.1056/NEJMoa1200388.

10. Liu P, Wang F, Ren S, Lin F, Yang Y, Fan X, et al. A propensity score analysis on the effect of on-pump versus off-pump coronary artery bypass grafting for patients with coronary artery disease. JThorac Dis. 2014;6(6):838-44. doi:10.3978/j.issn.2072-1439.2014.05.08.

11. Raja SG, Benedetto U. Off-pump coronary artery bypass grafting: misperceptions and misconceptions. World J Methodol. 2014;4(1):6-10. doi:10.5662/wjm.v4.i1.6.

12. Lima R, Diniz R, Césio A, Vasconcelos F, Gesteira M, Menezes A, et al. Myocardial revascularization in octogenarian patients: retrospective and comparative study between patients operated on pump and off pump. Braz J Cardiovasc Surg. 2005;20(1):8-13.

13. Sá MP, Lima LP, Rueda FG, Escobar RR, Cavalcanti PE, Thé EC, et al. Comparative study between on-pump and off-pump coronary artery bypass graft in women. Rev Bras Cir Cardiovasc. 2010;25(2):238-44. doi:10.1590/s0102-76382010000200018.

14. Cantero MA, Almeida RM, Galhardo R. Analysis of immediate results of on-pump versus off-pump coronary artery bypass grafting surgery. Rev Bras Cir Cardiovasc. 2012;27(1):38-44. doi:10.5935/1678-9741.20120007.

15. Brewer R, Theurer PF, Cogan CM, Bell GF, Prager RL, Paone G, et al. Morbidity but not mortality is decreased after off-pump coronary artery bypass surgery. Ann Thorac Surg. 2014;97(3):831-6.

16. Bakaeen FG, Kelly RF, Chu D, Jessen ME, Ward HB, Holman WL. Trends over time in the relative use and associated mortality of on-pump and off-pump coronary artery bypass grafting in the veterans affairs system. JAMA Surg. 2013;148(11):1031-6. doi:10.1001/jamasurg.2013.3580.

17. Deb S, Austin PC, Tu JV, Ko DT, Mazer CD, Kiss A, et al. A review of propensity-score methods and their use in cardiovascular research. Can J Cardiol. 2016;32(2):259-65. doi:10.1016/j.cjca.2015.05.015.

18. Kuss O, Blettner M, Börgermann J. Propensity score: an alternative

\section{Authors' Roles \& Responsibilities}

AR Substantial contributions to the conception or design of the work; or the acquisition, analysis, or interpretation of data for the work; drafting the work or revising it critically for important intellectual content; agreement to be accountable for all aspects of the work in ensuring that questions related to the accuracy or integrity of any part of the work are appropriately investigated and resolved; final approval of the version to be published

GC Substantial contributions to the conception or design of the work; or the acquisition, analysis, or interpretation of data for the work; final approval of the version to be published

PN Substantial contributions to the conception or design of the work; or the acquisition, analysis, or interpretation of data for the work; final approval of the version to be published

BSH Substantial contributions to the conception or design of the work; or the acquisition, analysis, or interpretation of data for the work; final approval of the version to be published

EL Substantial contributions to the conception or design of the work; or the acquisition, analysis, or interpretation of data for the work; final approval of the version to be published

MS Substantial contributions to the conception or design of the work; or the acquisition, analysis, or interpretation of data for the work; final approval of the version to be published

FLL Drafting the work or revising it critically for important intellectual content; final approval of the version to be published

FL Agreement to be accountable for all aspects of the work in ensuring that questions related to the accuracy or integrity of any part of the work are appropriately investigated and resolved; final approval of the version to be published method of analyzing treatment effects. Dtsch Arztebl Int. 2016;113(3536):597-603. doi:10.3238/arztebl.2016.0597.

19. Bakaeen FG, Chu D, Kelly RF, Holman WL, Jessen ME, Ward HB. Perioperative outcomes after on- and off-pump coronary artery bypass grafting. Tex Heart Inst J. 2014;41 (2):144-51. doi:10.14503/THIJ-13-3372.

20. Hannan EL, Wu C, Smith CR, Higgins RS, Carlson RE, Culliford AT, et al. Off-pump versus on-pump coronary artery bypass graft surgery: differences in short-term outcomes and in long-term mortality and need for subsequent revascularization. Circulation. 2007;1 16(10):114552. doi:10.1161/CIRCULATIONAHA.106.675595. 